\title{
The impact of endometrial thickness change after progesterone administration on pregnancy outcome in patients transferred with single frozen-thawed blastocyst
}

\author{
Zhiqin Bu ${ }^{\dagger}$, Xinhong Yang ${ }^{\dagger}$, Lin Song, Beijia Kang and Yingpu Sun ${ }^{*}$ (D)
}

\begin{abstract}
Background: The aim of this study was to explore the impact of endometrial thickness change after progesterone administration on pregnancy outcome in patients transferred with single frozen-thawed blastocyst.

Methods: This observational cohort study included a total of 3091 patients undergoing their first frozen-thawed embryo transfer (FET) cycles between April 2015 to March 2019. Endometrial thickness was measured by transvaginal ultrasound twice for each patient: on day of progesterone administration, and on day of embryo transfer. The change of endometrial thickness was recorded.

Results: Regardless of endometrial preparation protocol (estrogen-progesterone/natural cycle), female age, body mass index (BMI), and infertility diagnosis were comparable between patients with an increasing endometrium on day of embryo transfer and those without. However, clinical pregnancy rate increases with increasing ratio of endometrial thickness. Compared with patients with Non-increase endometrium, those with an increasing endometrium on day of embryo transfer resulted in significantly higher clinical pregnancy rate $(56.21 \%$ vs $47.13 \%$, $P=0.00$ in estrogen-progesterone cycle; $55.15 \%$ vs $49.55 \%, P=0.00$ in natural cycle).
\end{abstract}

Conclusions: In most patients, endometrial thickness on day of embryo transfer (after progesterone administration) increased or kept being stable compared with that on day of progesterone administration. An increased endometrium after progesterone administration was associated with better pregnancy outcome.

Keywords: Infertility, Endometrium, IVF/ICSI outcome

\section{Introduction}

For decades, the debate about the relationship between endometrial thickness and clinical pregnancy has never stopped [1, 2]. At present, most studies have observed that thin endometrium has an adverse effect on pregnancy outcome during in vitro fertilization (IVF) treatment, even though the specific mechanism is not well understood [3-5].

\footnotetext{
*Correspondence: syp2008@vip.sina.com

† Zhiqin Bu and Xinhong Yang contributed equally to this work. Reproductive Medical Center, Henan Province Key Laboratory for Reproduction and Genetics, The First Affiliated Hospital of Zhengzhou University, 1\# Jianshe East, Zhengzhou, Henan Province, China
}

Why scholars are keen to study the relationship between endometrium and pregnancy outcome? One of the important reasons is that endometrial thickness may represent endometrial receptivity [6]. Under physiological conditions, endometrial receptivity mainly refers to the ability of the endometrium to accept embryos when implantation window is open, which is around 7 days after ovulation in natural menstrual cycle. In patients undergoing IVF treatment, the day of embryo transfer is usually considered to be in the middle of implantation window. Thus, it is reasonable to believe that the condition of endometrium on day of transfer, but not on day of human chorionic gonadotropin (hCG) 
trigger or progesterone administration (both are at least 3-5 days before embryo transfer), is more representative of endometrial receptivity.

It is known that the condition of endometrium is changeable in natural menstrual cycle, and also in IVF treatment. One of the typical change is that endometrial pattern will be changed from pattern A (triple-line pattern)/pattern B (intermediate isoechogenic pattern) to pattern $\mathrm{C}$ (homogenous, hyperechogenic pattern) after hCG or progesterone administration during IVF cycles [7, 8], but little is known about the endometrial thickness change after hCG or progesterone administration. In the morning of embryo transfer, endometrial pattern and thickness of all patients are routinely re-evaluated by trans-vaginal ultrasound in our center. Thus it is convenient for us to record the change of endometrial thickness after progesterone administration in frozen-thawed embryo transfer (FET) cycles.

The aim of the current study was to record the dynamic change of endometrial thickness (Day of embryo transfer Versus. Day of progesterone administration), and to investigate the impact of endometrial thickness change on pregnancy outcomes in women with single blastocyst transfer in their first FET cycles.

\section{Materials and methods}

In 2015 we registered this observational cohort study on Clinical Trial (NCT02420704). In total, 3091 patients undergoing their first FET cycles $[1757$ Estrogenprogesterone (EP) cycles and 1334 Natural cycles (NC)] from April 2015 to March 2019 were included. Data in this study were from the Clinical Reproductive Medicine Management System/Electronic Medical Record Cohort Database (CCRM/EMRCD) in Reproductive Medical Center, First Affiliated Hospital of Zhengzhou University. This study has been approved by The Institutional Review Board of Zhengzhou University.

Only patients with single blastocyst transfer were included. Endometrial pattern was pattern A/B on day of progesterone administration, and was pattern $\mathrm{C}$ on day of embryo transfer for all patients.

For exclusion criteria, patients with low quality embryo (a blastocyst score $<3 \mathrm{BC}$ according to Gardner system) or with thin endometrial thickness $(<7 \mathrm{~mm}$ on day of progesterone administration) were also excluded. In addition, pre-implantation genetic diagnosis cycles, as well as oocyte donation cycles were also not included.

Preparation of endometrium for FET was EP and NC. The detailed protocol for endometrial preparation, endometrial pattern classification, and also the method of how to measure endometrial thickness, all have been described in our previous work [3].

\section{Endometrial thickness on day of progesterone administration}

For EP cycles, only oral estradiol ([progynova]; Bayer, Germany) were used in the first 12-14 days. Once endometrial thickness reached $\geq 7 \mathrm{~mm}$, progesterone in oil $(60 \mathrm{mg})$ was added. For NC cycles, progesterone in oil $(40 \mathrm{mg}$ ) was first added on the day of ovulation. Endometrial thickness on this progesterone administration day will be recorded by trans-vaginal ultrasound examination.

\section{Endometrial thickness on day of embryo transfer}

All FET patients in our center were hospitalized the day before embryo transfer as a routine practice. Endometrial thickness was re-evaluated on the morning of embryo transfer day, also by trans-vaginal ultrasound, to exclude cavity fluid and other unfavorable conditions.

After embryo transfer, luteal supplement was changed to vaginal progesterone gel $(90 \mathrm{mg}$, Crinone $8 \%$; Merck Serono) and oral dydrogesterone (20 mg duphaston; Abbott). Clinical pregnancy was confirmed by ultrasound observation 5 weeks after embryo transfer.

\section{Statistical analysis}

Patient with endometrial thickness on day of embryo transfer thicker than that on day of progesterone administration were allocated into the Increasing Group. The Non-increase Group included patients with endometrial thickness on day of embryo transfer equal to, or less than that on day of progesterone administration.

Student's t-test was used to detect difference between continuous variables, and chi-square test were for categorical variables. Statistical analysis was performed with SPSS (Statistical Package for Social Science, SPSS Inc., Chicago, IL, USA) version 19.0. A $P<0.05$ was considered to be statistically significant.

\section{Results}

In this observational cohort study, a total of 3091 first FET cycles with single blastocyst transfer were included. A brief description and flow chart of this study was shown in Fig. 1. In EP cycle, endometrial thickness on day of embryo transfer decreased in $19.63 \%$ patients, and this proportion was even higher (26.24\%) in NC cycle.

Table 1 shows the basic character and pregnancy outcomes in patients according to the change of endometrial thickness. Regardless of EP or NC cycle, female age, body mass index (BMI), and infertility diagnosis were comparable between those with an increasing endometrium on day of embryo transfer and those without. However, for both EP and NC cycles, endometrial thickness on day of progesterone administration in Increasing Group was significantly thinner at first $(9.03 \pm 1.34$ vs $9.61 \pm 1.57, P=0.00$ in EP cycle; $9.64 \pm 1.70$ vs $10.69 \pm$ 


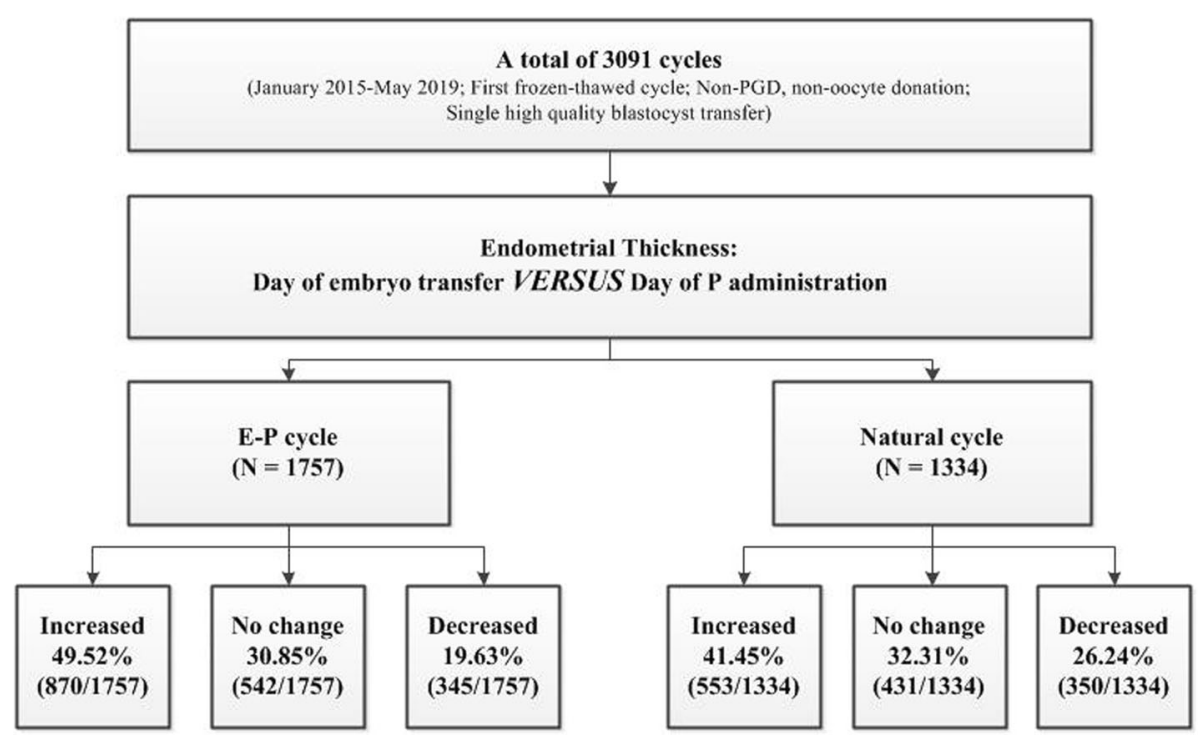

Fig. 1 Study flow chart. PGD preimplantation genetic diagnosis, $P$ progesterone, E-P estrogen-progesterone

$1.96, P=0.00$ in NC cycle); it increased dramatically at the end and became thicker on day of embryo transfer when compared with that in Non-increase Group $(10.60 \pm 1.66$ vs $9.11 \pm 1.49, P=0.00$ in EP cycle; $11.25 \pm$ 1.93 vs $10.03 \pm 1.82, P=0.00$ in NC cycle).

As for the pregnancy outcome, Fig. 2 clearly shows that clinical pregnancy rate increases with increasing ratio of endometrial thickness. Even though with limited participants, in endometrial thickness decreasing $\geq 20 \%$ group, clinical pregnancy rate in EP and NC cycles was only 37.14 and $32.35 \%$, respectively. However, in endometrial thickness increasing $\geq 20 \%$ group, clinical pregnancy rate was nearly $60 \%$ in both $\mathrm{EP}$ and NC cycles. In addition, Table 1 also shows that for patients either in EP or NC cycle group, compared with patients with Non-increase endometrium, those with an increasing endometrium on day of embryo transfer resulted in significantly higher clinical pregnancy rate $(56.21 \%$ vs $47.13 \%, P=0.00$ in EP cycle; $55.15 \%$ vs $49.55 \%, P=0.00$ in NC cycle). Moreover, early spontaneous abortion rate in Non-increase group was slightly higher even without statistical significant. Ectopic pregnancy rate was also comparable in Increasing group and Non-increase group irrespective of EP or NC cycles.

Table 1 Patient basic characteristic and pregnancy outcomes in women transferred with single frozen thawed blastocyst

\begin{tabular}{|c|c|c|c|c|c|c|}
\hline & \multicolumn{3}{|c|}{ Estrogen-progesterone cycle $(N=1757)$} & \multicolumn{3}{|c|}{ Natural cycle $(N=1334)$} \\
\hline & $\begin{array}{l}\text { Increasing } \\
(N=870)\end{array}$ & $\begin{array}{l}\text { Non-increase } \\
(N=887)\end{array}$ & $P$ & $\begin{array}{l}\text { Increasing } \\
(N=553)\end{array}$ & $\begin{array}{l}\text { Non-increase } \\
(N=781)\end{array}$ & $P$ \\
\hline Age (years) & $30.27 \pm 4.57$ & $30.63 \pm 4.98$ & 0.11 & $31.31 \pm 4.64$ & $31.76 \pm 4.96$ & 0.10 \\
\hline Body Mass Index $\left(\mathrm{Kg} / \mathrm{m}^{2}\right)$ & $22.67 \pm 3.22$ & $22.73 \pm 3.27$ & 0.71 & $22.64 \pm 3.23$ & $22.65 \pm 3.19$ & 0.97 \\
\hline History of prior gravidity (\%) & $55.17 \%(480 / 870)$ & $56.37 \%(500 / 887)$ & 0.61 & $67.09 \%(371 / 553)$ & $63.38 \%(495 / 781)$ & 0.16 \\
\hline \multicolumn{7}{|l|}{ Infertility diagnosis } \\
\hline Tubal factor & $36.32 \%(316 / 870)$ & $36.30 \%(322 / 887)$ & & $51.72 \%(286 / 553)$ & $52.75 \%(412 / 781)$ & \\
\hline Ovulation disorder & $26.78 \%(233 / 870)$ & $27.96 \%(248 / 887)$ & 0.75 & - & - & 0.94 \\
\hline Male factor & $21.49 \%(187 / 870)$ & $18.49 \%(164 / 887)$ & & $3.15 \%(128 / 553)$ & $23.30 \%(182 / 781)$ & \\
\hline Combined and others & $15.40 \%(134 / 870)$ & $17.25 \%(153 / 887)$ & & $25.14 \%(139 / 553)$ & $23.94 \%(187 / 781)$ & \\
\hline Endometrial thickness: starting progesterone & $9.03 \pm 1.34$ & $9.61 \pm 1.57$ & 0.00 & $9.64 \pm 1.70$ & $10.69 \pm 1.96$ & 0.00 \\
\hline Endometrial thickness: embryo transfer & $10.60 \pm 1.66$ & $9.11 \pm 1.49$ & 0.00 & $11.25 \pm 1.93$ & $10.03 \pm 1.82$ & 0.00 \\
\hline Clinical pregnancy rate (\%) & $56.21 \%(489 / 870)$ & $47.13 \%(418 / 887)$ & 0.00 & $55.15 \%(305 / 553)$ & $49.55 \%(387 / 781)$ & 0.04 \\
\hline Ectopic pregnancy rate (\%) & $1.43 \%(7 / 489)$ & $2.39 \%(10 / 418)$ & 0.29 & $1.64 \%(5 / 305)$ & $1.55 \%(6 / 387)$ & 0.93 \\
\hline Early spontaneous abortion rate (\%) & $14.1 \%(69 / 489)$ & $18.66 \%(78 / 418)$ & 0.06 & $12.77 \%(40 / 305)$ & $13.70 \%(53 / 387)$ & 0.82 \\
\hline
\end{tabular}




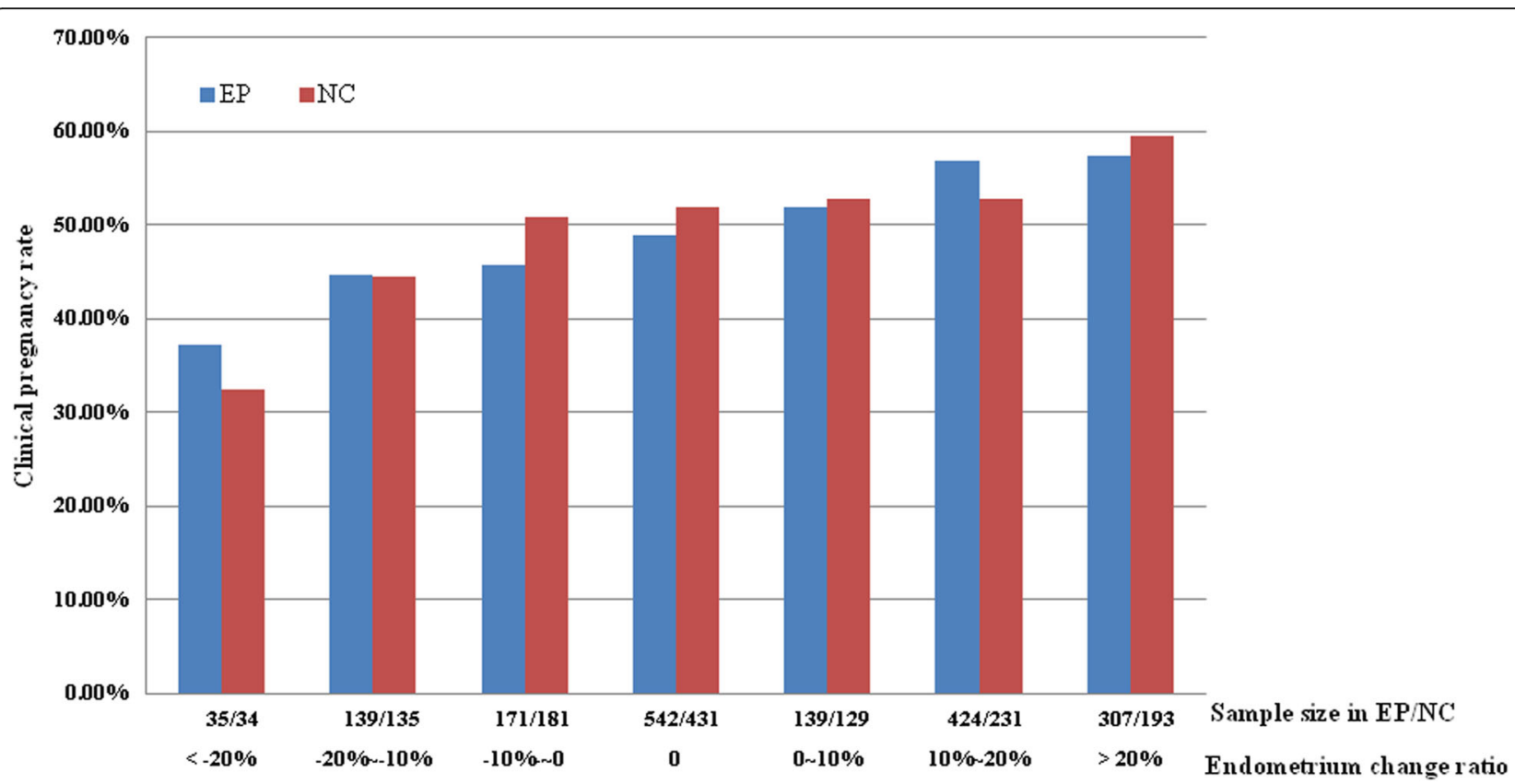

Fig. 2 Relationship between endometrium change ratio and clinical pregnancy rate. EP estrogen-progesterone, NC natural cycle

\section{Discussion}

To date, numerous studies, including our previous work, have focused on the impact of endometrial thickness on IVF outcome, and the conclusions are basically the same: a thin endometrium $(<7 \mathrm{~mm}$ in most cases $)$ is predicable for a poor pregnancy outcome $[9,10]$. Recently, a cohort study including over 40,000 participants from Canada, and another UK study with over 20,000 cycles both showed similar results $[11,12]$.

In most previous studies, endometrial thickness was measured during ovarian stimulation (in fresh embryo transfer cycles), or during proliferation phase before progesterone administration (in frozen thawed embryo transfer cycles), both are several days ahead of embryo transfer. However, in 2009 Barker et al have noticed this issue and evaluated the relationship between endometrial thickness in luteal phase (day of ET), in the late follicular phase, and pregnancy outcome in oocyte donation model [13]. Data from this study showed that endometrial thickness in recipients prior to and following progesterone had no impact on pregnancy outcomes. More recently, another cohort study including 271 FET cycles also evaluated endometrial thickness on both the end of the estrogen phase and the day of embryo transfer [14]. Interestingly, they found that a decreased endometrium (endometrial compaction) in response to progesterone results in better pregnancy outcome in FET cycles, which means that the greater the degree of compaction, the higher the ongoing pregnancy rate. In Barker's study, endometrial thickness in pregnant and non-pregnant group after progesterone administration increased by 1.3 and $0.7 \mathrm{~mm}$, respectively. Our study also showed that in 3091 cycles, endometrial thickness on day of embryo transfer increased by $0.41 \mathrm{~mm}$ on average compared with the day of progesterone administration(from $9.73 \mathrm{~mm}$ to $10.14 \mathrm{~mm}$ ). In addition, the ratio of patients with endometrial compaction $>5 \%$ was $21.96 \%(679 / 3091)$ in our study, however, this is far less than that $(42.44 \%=115 / 271)$ from Haas's study.

Question is, how does the endometrial thickness change during the proliferation phase and the luteal phase under physiological conditions? In natural menstrual cycle, estrogen and progesterone secreted by the corpus luteum makes the endometrium from the proliferative phase continue to grow. Endometrial gland is more curved, and secretion occurs. Meanwhile, blood vessels rapidly increased, and endometrial stroma became loose. It was shown that endometrial thickness increase during the follicular phase of the menstrual cycle, peak prior to ovulation, plateau during the early luteal phase and then decline prior to menstruation [15]. Thus, an increased endometrium after progesterone administration in FET cycles seemed to be a reasonable phenomenon, and we speculated that pregnancy was much easier in patients with an increased endometrium, which is consistent with results from our study.

In addition, during FET procedure, estrogen level may differ between $\mathrm{NC}$ cycles and EP cycles after progesterone administration. In EP cycles, oral estradiol is continued after progesterone administration; however, in NC cycles, only progesterone was used after ovulation without any estrogen supplementation. It is reasonable to 
Table 2 Endometrial thickness on day of progesterone administration/embryo transfer according endometrial change ratio

\begin{tabular}{lllllllll}
\hline & Endometrial change ratio & $<-20 \%$ & $-20 \% \sim-10 \%$ & $-10 \%-\sim ~$ & 0 & $0 \sim 10 \%$ & $10 \% \sim 20 \%$ & $>20 \%$ \\
\hline EP cycle & No. & 35 & 139 & 171 & 542 & 139 & 424 & 307 \\
& Age (year) & 30.77 & 30.61 & 30.68 & 30.61 & 30.55 & 30.3 & 30.11 \\
& Thickness on dPA (mm) & 10.8 & 9.66 & 10.22 & 9.33 & 9.96 & 8.92 & 8.77 \\
& Thickness on dET (mm) & 7.96 & 8.25 & 9.36 & 9.33 & 10.69 & 10.01 & 11.36 \\
\multirow{5}{*}{ NC cycle } & No. & 34 & 135 & 181 & 431 & 129 & 231 & 193 \\
& Age (year) & 31.44 & 32.05 & 31.83 & 31.66 & 31.21 & 31.16 & 31.59 \\
& Thickness on dPA (mm) & 12.76 & 10.88 & 11.07 & 10.31 & 11.04 & 9.23 & 9.19 \\
& Thickness on dET (mm) & 9.28 & 9.17 & 10.14 & 10.31 & 11.91 & 10.38 \\
\hline
\end{tabular}

$E P$ estrogen-progesterone, NC natural cycle, $d P A$ day of progesterone administration, $d E T$ day of embryo transfer; Endometrial change ratio $=($ Thickness on dETThickness on dPA)/ Thickness on dPA* $100 \%$

find that endometrial thickness increased $0.52 \mathrm{~mm}$ on average in EP cycles, but it only increased $0.28 \mathrm{~mm}$ on average in $\mathrm{NC}$ cycles from our study.

Even though as an observational cohort study, our large sample size study indeed has several strengths. First, only high quality, single blastocyst transfer cycles were included, because number of embryo and quality of embryo are very important factors related with pregnancy outcome in IVF treatment cycles. Second, patients with thin endometrium $(<7 \mathrm{~mm})$ was excluded as situation of this group of patients was complicated. In addition, we included patients in FET transfer with both EP and NC cycles. Patients in our center with single blastocyst transfer in fresh embryo transfer cycles are high ovarian responders. Ovarian stimulation protocols are different, and are out of control for further analysis. Another point is that the endometrial situation of each patients is re-evaluated on the morning of embryo transfer day also by trans-vaginal ultrasonic examination, which is a more accurate way to measure endometrial thickness. In Haas's study, endometrial thickness was measured by means by pelvic (transabdominal) ultrasound [14]. This may explain the controversy between the totally opposite results from these two studies.

Table 2 briefly shows the endometrial thickness on day of progesterone, on day of embryo transfer, in patients with different endometrial thickness change group. It warn us endometrial thickness examination after progesterone administration is important. Patients with dramatic decrease in endometrial thickness do have good endometrium at first, but they do not result in final optimistic outcome.

However, several limitations exist in this study. First, the specific molecular and cellular evident behind this phenomenon needs to be explored in future studies. In addition, as a preliminary observational study, no additional clinical management was performed if endometrium did not increase, because endometiral thickness was $\geq 7 \mathrm{~mm}$ in these cases. Moreover, even each physician in our center are well-trained, and are capable of ultrasonic examination under Standard Operation Procedure, inter-observer variability for endometrial thickness may bring some bias to this study.

\section{Conclusion}

In summary, the current large sample size observational cohort study demonstrated the dynamic change of endometrial thickness after progesterone administration in FET cycles. In both EP and NC cycles for endometrium preparation, endometrial thickness on day of embryo transfer increased or kept being stable compared with that on day of progesterone administration in most patients. In addition, an increased endometrium after progesterone administration was associated with better pregnancy outcome. Interestingly, clinical pregnancy outcomes and the increasing rate of endometrium were positively correlated.

\section{Abbreviations}

BMI: Body mass index; EP: Estrogen-progesterone; FET: Frozen thawed embryo transfer; hCG: Human chorionic gonadotropin; ICSI: Intracytoplasmic sperm injection; IVF: In ivtro fertilization; NC: Natural cycle

\section{Acknowledgements}

The authors would like to thank Dr. Keyan Wang from School of Public Health, Zhengzhou University, for her assistance in data analysis.

\section{Authors' contributions}

YS, ZB and XY contributed to the study design, data analysis and manuscript preparation. LS and BK handled patient recruitment and data collection. All authors read and approved the final manuscript.

\section{Funding}

This work was supported by National Natural Science Foundation of China (No. 81801448 to ZQ.B, and No. 81820108016 to YP.S).

Availability of data and materials

All data supporting the conclusion of this article are included.

Ethics approval and consent to participate

The study has received approval and was carried out in accordance with the approved guidelines from the Zhengzhou University Research Ethics Board.

Consent for publication

Not applicable. 


\section{Competing interests}

The authors declare that they have no competing interests.

Received: 17 September 2019 Accepted: 18 November 2019

Published online: 25 November 2019

\section{References}

1. Kasius A, Smit JG, Torrance HL, Eijkemans MJ, Mol BW, Opmeer BC, Broekmans FJ. Endometrial thickness and pregnancy rates after IVF: a systematic review and meta-analysis. Hum Reprod Update. 2014;20:530-41.

2. Griesinger G, Trevisan S, Cometti B. Endometrial thickness on the day of embryo transfer is a poor predictor of IVF treatment outcome. Hum Reprod Open. 2018;2018:x31.

3. Bu Z, Wang K, Dai W, Sun Y. Endometrial thickness significantly affects clinical pregnancy and live birth rates in frozen-thawed embryo transfer cycles. Gynecol Endocrinol. 2016;32:524-8.

4. Oron G, Hiersch L, Rona S, Prag-Rosenberg R, Sapir O, Tuttnauer-Hamburger M, Shufaro Y, Fisch B, Ben-Haroush A. Endometrial thickness of less than 7.5 $\mathrm{mm}$ is associated with obstetric complications in fresh IVF cycles: a retrospective cohort study. Reprod BioMed Online. 2018;37:341-8.

5. Shi W, Zhang S, Zhao W, Xia X, Wang M, Wang H, Bai H, Shi J. Factors related to clinical pregnancy after vitrified-warmed embryo transfer: a retrospective and multivariate logistic regression analysis of 2313 transfer cycles. Hum Reprod. 2013;28:1768-75.

6. Craciunas L, Gallos I, Chu J, Bourne T, Quenby S, Brosens JJ, Coomarasamy A. Conventional and modern markers of endometrial receptivity: a systematic review and meta-analysis. Hum Reprod Update. 2019;25:202-23.

7. Zhao J, Zhang Q, Wang Y, Li Y. Endometrial pattern, thickness and growth in predicting pregnancy outcome following 3319 IVF cycle. Reprod BioMed Online. 2014;29:291-8.

8. Yang W, Zhang T, Li Z, Ren X, Huang B, Zhu G, Jin L. Combined analysis of endometrial thickness and pattern in predicting clinical outcomes of frozen embryo transfer cycles with morphological good-quality blastocyst: a retrospective cohort study. Medicine (Baltimore). 2018;97:e9577.

9. Bu Z, Sun Y. The impact of endometrial thickness on the day of human chorionic Gonadotrophin (hCG) administration on ongoing pregnancy rate in patients with different ovarian response. PLoS One. 2015;10:e145703.

10. Chen SL, Wu FR, Luo C, Chen X, Shi XY, Zheng HY, Ni YP. Combined analysis of endometrial thickness and pattern in predicting outcome of in vitro fertilization and embryo transfer: a retrospective cohort study. Reprod Biol Endocrinol. 2010;8:30.

11. Liu K, Hartman M, Hartman A, Luo Z, Mahutte N. The impact of a thin endometrial lining on fresh and frozen-thaw IVF outcomes: an analysis of over 40000 embryo transfers. Hum Reprod. 2018;33:1883-8.

12. Gallos I, Khairy M, Chu J, Rajkhowa M, Tobias A, Campbell A, Dowell K, Fishel S, Coomarasamy A. Optimal endometrial thickness to maximize live births and minimize pregnancy losses: analysis of 25,767 fresh embryo transfers. Reprod BioMed Online. 2018;37:542-8.

13. Barker MA, Boehnlein LM, Kovacs P, Lindheim SR. Follicular and luteal phase endometrial thickness and echogenic pattern and pregnancy outcome in oocyte donation cycles. J Assist Reprod Genet. 2009;26:243-9.

14. Haas J, Smith R, Zilberberg E, Nayot D, Meriano J, Barzilay E, Casper RF. Endometrial compaction (decreased thickness) in response to progesterone results in optimal pregnancy outcome in frozen-thawed embryo transfers. Fertil Steril. 2019;112:503-9.

15. Wang ZM. Transvaginal ultrasonographic monitoring on the morphological changes of ovary and endometrium during normal menstrual cycle. Zhonghua Fu Chan Ke Za Zhi. 1993;28:18-20.

\section{Publisher's Note}

Springer Nature remains neutral with regard to jurisdictional claims in published maps and institutional affiliations.

Ready to submit your research? Choose BMC and benefit from:
- fast, convenient online submission
- thorough peer review by experienced researchers in your field
- rapid publication on acceptance
- support for research data, including large and complex data types
- gold Open Access which fosters wider collaboration and increased citations
- maximum visibility for your research: over 100M website views per year
At BMC, research is always in progress.
Learn more biomedcentral.com/submissions

